\title{
Image Guided Tone Mapping with Locally Nonlinear Model
}

\author{
Huxiang Gu, Ying Wang, Shiming Xiang, Gaofeng Meng, and Chunhong Pan \\ Institute of Automation, Chinese Academy of Science \\ $\{\mathrm{hxgu}, \mathrm{ywang}$, smxiang, gfmeng, chpan $\}$ @nlpr.ia.ac.cn
}

\begin{abstract}
In this paper, we propose an effective locally nonlinear tone mapping algorithm for compressing the High Dynamic Range (HDR) images. Instead of linearly scaling the luminance of pixels, our core idea is to introduce local gamma correction with adaptive parameters on small overlapping patches over the entire input image. A framework for HDR image compression is then introduced, in which the global optimization problem is deduced and two guided images are adopted to induct the optimum solution. The optimal compression can finally be achieved by solving the optimization problem which can be transformed to a sparse linear equation. Extensive experimental results on a variety of HDR images and a carefully designed perceptually evaluation have demonstrated that our approach can achieve better performances than the state-of-theart approaches.
\end{abstract}

Keywords: high dynamic range, tone mapping, locally nonlinear model, guided image.

\section{Introduction}

HDR images can capture greater dynamic range of real world scenes than LDR images by using 16 bit or even higher bits with floating point type. This wide dynamic range allows HDR images to more accurately represent the intensity levels in real world. Unfortunately, most of the modern display devices have limited dynamic range. Hence, a number of tone mapping operators have been proposed to compress the high dynamic range of HDR images to the displayable range while preserve the visual contents[1 3$]$. These tone mapping operators are useful not only for HDR photography but also for lighting simulation in realistic rendering [4]. Therefore, the last three decades enjoy a boom of tone mapping algorithms in both the computational photography community and computer vision community [5, 4,6 , 8 ].

In literature, the tone mapping operators can be roughly classified into two categories: global operators and local operators. Global operators [9 13] can be regarded as spatially uniform methods because the same mapping function is used for all the pixels of the input HDR image. They are simple and fast. However, they suffer from losing visual details in both bright areas and dark regions because they compress all the structures and details without considering

A. Fitzgibbon et al. (Eds.): ECCV 2012, Part IV, LNCS 7575, pp. 786-799, 2012.

(C) Springer-Verlag Berlin Heidelberg 2012 
the local luminance variation. Therefore a variety of local operators which model spatial adaptation by using locally changing functions have been proposed to compress the dynamic range while maintain or enhance the details $6,14,15,2$, 3]. Most local operators decompose the input HDR image into different layers 1] or areas [16]. Different mapping functions for each layer or area are adopted to compress the dynamic range and final results are achieved by a combination of these layers or scales after contrast reduction. Most of these local operators suffer from halo effects which are critical in HDR images. Then several operators have been proposed to improve this flaw [15, 2, 14, 3].

Although many excellent tone mapping operators have been proposed, tone mapping algorithm is still far beyond perfection. None of the present approaches have met the most challenging goal that an ideal tone mapping operator should achieve perceptually natural LDR images with precise details as well as free of any kinds of distortions or halo effect.

The main purpose of this paper is to introduce an effective tone mapping operator which can achieve perceptually pleasing results with fine details. The output LDR image has a high contrast and free of distortions or halo effects. Instead of linearly scaling the luminance of pixels, we propose a new tone mapping algorithm based on local gamma correction with adaptive parameters. Our method is based on the Weber-Fechner Law [17] that the human eye's subjective perception of brightness is related to the physical stimulation of light intensity in a manner which is similar with the power function used for gamma correction.

Our method benefits from the following two main contributions:

(1). An effective locally nonlinear model based on the Weber-Fechner Law 17] is proposed. Our model coincides with the nonlinear relationship between the physical magnitudes of stimuli and the perceived intensity of the stimuli. Compared with the locally linear model [8], our model has not only a more reasonable physical explanation, but also a wider applicability.

(2). When solving our locally nonlinear model, we add two constraint items into our energy function to avoid distortions and then achieve perceptually pleasing LDR images. Two guided images are creatively adopted in these two constraint items. These two guided images are critical for a natural LDR image which has fine details but no distortions.

\section{Previous Work}

Because of the great advantages over LDR images [4, 7], HDR images as well as tone mapping algorithms are therefore drawing a world of excellent researchers' attention [4, 15, 2, 3, 5, 1, 6].

Debevec and Malik [4] proposed that a HDR image can be created from three or more LDR images of the same scene under different exposures. With the development of photograph technology, we can get access to HDR cameras which can take HDR photos and videos directly now. Therefore, there is an increasing demand for tone mapping algorithms. These tone mapping algorithms can be roughly classified into two categories: global operators and local operators. 
The global tone mapping methods are simply mapped the input HDR image $I^{h}(x, y)$ to an output LDR image $I^{l}(x, y)=f\left(I^{h}(x, y)\right)$, where $f()$ is a global compression function which is spatially invariant, such as linear function, gamma function [18], histogram based function [10] and the function adapted to tone reproduction curves [12]. These methods are simple and fast, but always fail in balancing between unveiling visual contents and preserving details.

Hence, local tone mapping methods are the recent literature to compress the high dynamic range while maintain or enhance the details. Most of the local tone mapping methods decompose the input HDR image into several layers or areas, apply different compressing algorithm in different layers or areas and recombine all the layers or areas into a LDR image. Similar with Durand 6], Farbman 14] decomposed the HDR image into a base layer and a detail layer, while the base layer is obtained by an alternative edge-reserving smoothing operator and the detail layer is got by subtracting the base layer from the input HDR image. Recently, Lee [16] segmented the input HDR image into different parts using K-means algorithm and then applied automatic gamma correction in different parts. However, how to appropriately deal with the scale decomposition, layer separation or image segmentation is an another difficult problem. Besides, these methods have a reputation of causing halo artifacts.

Later, Li [2] improved the condition of halo artifacts by using a symmetrical analysis-synthesis filter bank and applying local gain control to the sub-bands. Results illustrated that the method of $\mathrm{Li}$ can achieve more satisfactorily than other multi-scale based methods. An alternative approach was also proposed by Fattal [15]. In their framework, the gradient field of the luminance image is manipulated by attenuating the magnitudes of large gradients as well as magnifying the small ones. Satisfactory results can be finally achieved by solving a Poisson equation. This gradient domain method is good at preserving fine details in dark regions and avoiding common artifacts.

More recently, Shan [8] provided us with a totally new tone mapping operator that performs locally linear adjustments on small overlapping windows over the entire input HDR image. They cast the compression task as a global optimization problem and achieve an optimal solution by solving a sparse equation. Locally linear method can effectively suppress local high contrast even in challenging HDR images. However, this method fails when luminance value of a local patch changes abruptly. An another impressive tone mapping method is the Local Laplacian filters [3]. Paris proposed a set of image filters using standard Laplacian pyramids to achieve edge-aware tone mapping. Local Laplacian filters can produce consistently high-quality results, especially in details manipulation. However, the complexity of this algorithm is a little high. Another imperfect point is that high-frequency textures are amplified by their detail-enhancing filter so that their result does not have a natural appearance.

As analyzed above, all these local operators firstly define a local measurement and then find a simple mapping function such as linear scaling. Although these tone mapping operators have achieved great success in many cases, the ideal target is still far away. 


\section{Motivation and Model}

\subsection{Motivation}

We are motivated mainly by the following facts. On the one hand, the WeberFechner Law [17] states that subjective sensation is proportional to the logarithm of the stimulus intensity. Compared with LDR image, HDR image can more precisely model the illumination variation in the real world. Thus it implies that the relationship between the input HDR image and the desired output LDR image is nonlinear and can be represented in a manner that is similar to the power function used for gamma correction. On the other hand, locally linear hypothesis which achieves great success in LDR image can not guarantee that it is still reasonable in HDR image because luminance value of a HDR image may vary a lot even in a local patch. The existing locally linear operator [8] (see Figure 1(a)) abruptly adopts locally linear hypothesis and therefore causes some distortions when a patch is bright enough or contains both dark and bright pixels. For instance, when a patch contains both dark and bright pixels (see Figure 1(c)), the dynamic range of the dark pixels will be compressed at the same rate as the bright ones, which results in losing details in this patch, as shown in Figure 2(c) and Figure 3(d).

Hence, a new locally nonlinear operator is needed to compress the contrast of a local patch as well as enhance the visual contents, even when luminance in this patch changes abruptly. Local gamma correction with adaptive parameters is therefore proposed to meet this demand, as shown in Figure 1(b). Extensive experimental results on a variety of HDR images have demonstrated the correctness of our motivation.

\subsection{Model}

In this part, we introduce our local gamma correction model. Given an input HDR image with radiance map $\mathbf{I}$, we compute the radiance map $\mathbf{J}$ of the output LDR image through $\mathbf{J}=f(\mathbf{I})$, where $f(\cdot)$ is a local compression function which should satisfy the local monotonic constraint. Considering a local patch $\Omega_{t}$ centering at pixel $t$, the local gamma correction model is

$$
\mathbf{j}(i)=\alpha_{t} \mathbf{i}(i)^{\beta_{t}} \quad i \in \Omega_{t},
$$

where $\mathbf{j}$ is a vector of luminance values in the local patch $\Omega_{t}$ of the output LDR image $\mathbf{J}$ and $\mathbf{i}$ is a vector of luminance values in the same local patch of the input HDR image $\mathbf{I}$. $\mathbf{j}(i)$ denotes the $i$-th element of $\mathbf{j}$ and the same with $\mathbf{i}(i)$. Parameter $\alpha_{t}$ and $\beta_{t}$ are constant values in each local patch, while $\alpha_{t}$ denotes the multiplier and $\beta_{t}$ is the index value. From the image's perspective, $\alpha$ and $\beta$ are named guided images in this paper, as shown in Figure 3(e) and Figure 3(f) respectively, which we will introduce in details in Section 4.3 .

Note that the locally linear model is a particular case of our locally nonlinear model. Actually, when parameter $\beta$ equals 1 , our model turns into the locally linear model. 


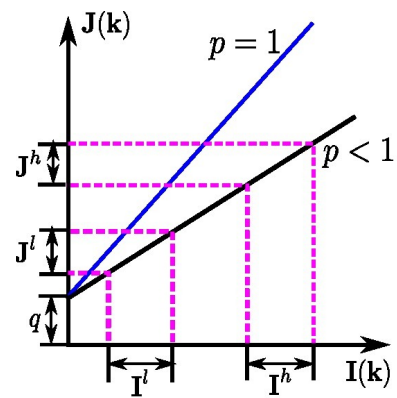

(a)

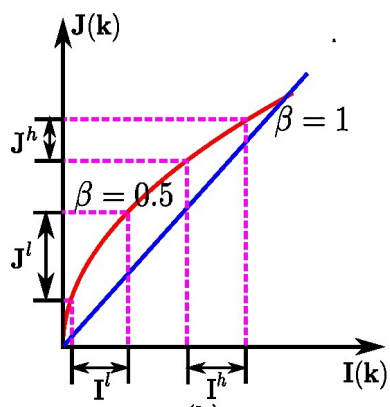

(b)

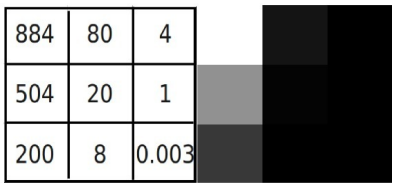

(c)

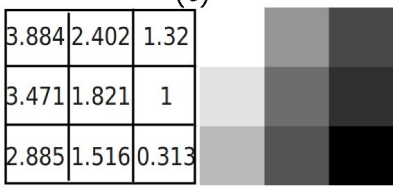

(d)

Fig. 1. Illustration of the locally linear model and our locally nonlinear model. (a) the locally linear model. (b) locally nonlinear model. (c) a local patch of the input HDR image, the left are relevant digital numbers and the right are luminance values. (d) the output local patch after tone mapping using our locally nonlinear method.

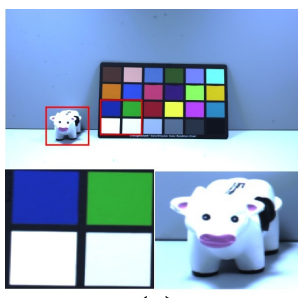

(a)

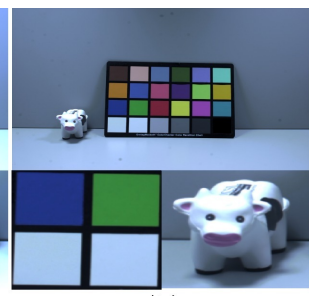

(b)

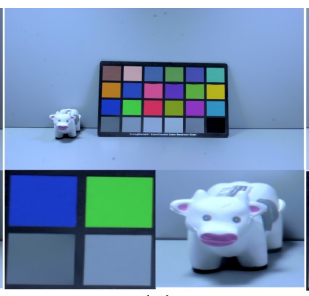

(c)

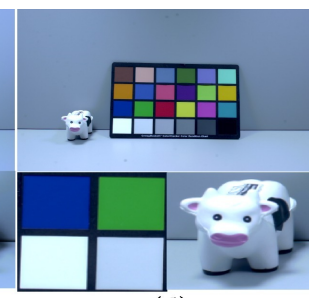

(d)

Fig. 2. Comparisons with the locally linear model [8]. (a) input HDR image. (b) result of $\operatorname{Shan}\left(\beta_{1}=0.7\right)$. (c) result of $\operatorname{Shan}\left(\beta_{1}=0.9\right)$. (d) our result.

Following we illustrate the advantage of our locally nonlinear model over the locally linear model in details. Instead of linearly compressing the contrast of a patch in an input HDR image, we adopt the local gamma correction strategy which can not only effectively compress the bright pixels but also enhance the dark ones in one hit even in challenging patches, as shown in Figure 1(d) and the sky-leaves part in Figure 3(h). The locally linear model usually fails when luminance value of a local patch changes abruptly, as shown in Figure 2(d) and Figure 5(c). In this situation, the dark pixels will turn bright because of $q($ intercept item of local linear model [8]) which determines the base radiance level (see Figure 1(a)). That is the reason why we adopt a new constraint item of $q$. Meanwhile, the dynamic range of the dark region will be compressed at the same rate as the bright pixels, which results in losing details in this patch. These flaws can be witnessed in the black area of the cow in Figure 22(c) and the sky-leaves in Figure 3(d). That is another reason why we introduce locally nonlinear model instead of locally linear model. 


\section{Algorithm and Implementation}

\subsection{Model Transformation}

When dealing with nonlinear model, we generally transform them to another domain in which nonlinear model turns to be linear model. After applying logarithmic transformation on both sides of Eq. (11), we get:

$$
\log \mathbf{j}(i)=\beta_{t} \log \mathbf{i}(i)+\log \alpha_{t} \quad i \in \Omega_{t} .
$$

Set $\mathbf{y}(i)=\log \mathbf{j}(i), \mathbf{x}(i)=\log \mathbf{i}(i), w_{t}=\beta_{t}, b_{t}=\log \alpha_{t}$, we get

$$
\mathbf{y}(i)=w_{t} \mathbf{x}(i)+b_{t} \quad i \in \Omega_{t} .
$$

Comparing Eq. (3) with Eq. (11), we find that locally nonlinear model in image domain is equivalent to linear compression in logarithmic domain.

\subsection{Model Solution}

The most common way to solve the parameters of linear regression problem can be described as

$$
\min _{w_{t}, b_{t}} \sum_{i \in \Omega_{t}}\left(\left\|\mathbf{y}(i)-w_{t} \mathbf{x}(i)-b_{t}\right\|^{2}+\lambda\left\|w_{t}\right\|^{2}\right) .
$$

However, the optimal solution of problem (4) are not so good, as shown in Figure 3(b). Therefore, inspired by [8], we adopt some prior information which are presented as the guided images to guide parameter $w_{t}$ and $b_{t}$. In order to get no distortion results, we add a new constraint item in which $b^{*}$ (namely the $\alpha$ image) is adopted to constrain the variation of parameter $b_{t}$. We also introduce a new approach to calculate $w^{*}$ (namely the $\beta$ image) to guide parameter $w_{t}$. These two guided images will be discussed in details in Section 4.3. As a result, the question now turns into minimize the local regression error $e_{t}$ as follows

$$
\min _{w_{t}, b_{t}} e_{t},
$$

where

$$
\left.e_{t}=\left\|\mathbf{y}_{\mathbf{t}}-w_{t} \mathbf{x}_{\mathbf{t}}-b_{t}\right\|^{2}+\lambda_{t}\left\|w_{t}-w_{t}^{*}\right\|^{2}+\tau_{t}\left\|b_{t}-b_{t}^{*}\right\|^{2}\right) .
$$

Here $\lambda_{t}=\mu w_{t}^{*-2}$ and $\tau_{t}=\nu b_{t}^{*-2}$ are regularization parameters in which $\mu=$ $\nu=0.1$. Denote $\mathbf{y}_{\mathbf{t}}=[y(1), y(2), \ldots, y(K)]^{T}$ and $\mathbf{x}_{\mathbf{t}}=[x(1), x(2), \ldots, x(K)]$ in which $K$ is the pixel number in each window. Extend $\mathbf{x}_{\mathbf{t}}=\left[\mathbf{x}_{\mathbf{t}} ; \mathbf{1}\right] \in \mathbf{R}^{2 \times K}$,

$$
\begin{gathered}
\mathbf{w}_{\mathbf{t}}=\left[w_{t}, b_{t}\right]^{T} \in \mathbf{R}^{2 \times 1}, \mathbf{w}_{\mathbf{t}}^{*}=\left[w_{t}^{*}, b_{t}^{*}\right]^{T} \in \mathbf{R}^{2 \times 1}, \mathbf{D}_{\mathbf{t}}=\left[\begin{array}{c}
\lambda_{t}, 0 \\
0, \tau_{t}
\end{array}\right] \in \mathbf{R}^{2 \times 2}, \text { we get } \\
e_{t}=\left\|\mathbf{x}_{\mathbf{t}}^{T} \mathbf{w}_{\mathbf{t}}-\mathbf{y}_{\mathbf{t}}\right\|^{2}+\frac{1}{2}\left(\mathbf{w}_{\mathbf{t}}-\mathbf{w}_{\mathbf{t}}^{*}\right)^{T} \mathbf{D}_{\mathbf{t}}\left(\mathbf{w}_{\mathbf{t}}-\mathbf{w}_{\mathbf{t}}^{*}\right) .
\end{gathered}
$$

The second term in Equation (77) is a variation of typical manifold regularization [19]. Similar with classical optimization of manifold learning, we can solve 
Equation (7) in derivation or iterative forms. Here we adopt the derivation form [20]. By taking the partial derivatives of $e_{t}$ with respect to $\mathbf{w}_{\mathbf{t}}$ and setting it to zero, we have

$$
\mathbf{w}_{\mathbf{t}}=\left(\mathbf{x}_{\mathbf{t}} \mathbf{x}_{\mathbf{t}}^{T}+\mathbf{D}_{\mathbf{t}}\right)^{-1}\left(\mathbf{x}_{\mathbf{t}} \mathbf{y}_{\mathbf{t}}+\mathbf{D}_{\mathbf{t}} \mathbf{w}_{\mathbf{t}}^{*}\right),
$$

Substituting Eq. (8) into (7) and then taking the partial derivatives of $e_{t}$ with respect to $\mathbf{y}_{\mathbf{t}}$, we can get

$$
\frac{d e_{t}}{d \mathbf{y}_{\mathbf{t}}}=\left(\mathbf{I}_{\mathbf{t}}-\mathbf{x}_{\mathbf{t}}^{T}\left(\mathbf{x}_{\mathbf{t}} \mathbf{x}_{\mathbf{t}}^{T}+\mathbf{D}_{\mathbf{t}}\right)^{-1} \mathbf{x}_{\mathbf{t}}\right) \mathbf{y}_{\mathbf{t}}-\mathbf{x}_{\mathbf{t}}^{T}\left(\mathbf{x}_{\mathbf{t}} \mathbf{x}_{\mathbf{t}}^{T}+\mathbf{D}_{\mathbf{t}}\right)^{-1} \mathbf{D}_{\mathbf{t}} \mathbf{w}_{\mathbf{t}}^{*},
$$

where $\mathbf{I}_{\mathbf{t}} \in \mathbf{R}^{K \times K}$ is an identity matrix. Then the total regression error of the input HDR image can be evaluated as

$$
E(\mathbf{Y})=\sum_{t} \mathbf{e}_{\mathbf{t}}
$$

Note that $\mathbf{y}_{\mathbf{t}}$ is just a subvector of the target LDR luminance image $\mathbf{Y}$. Define a selection matrix $\mathbf{S}_{\mathbf{t}} \in \mathbf{R}^{K \times N}$ ( $N$ is the total number of pixels in the input HDR image) as

$$
\mathbf{S}_{\mathbf{t}}(i, j)= \begin{cases}1 & \text { if } \mathbf{y}_{\mathbf{t}}(i) \text { is the } j \text {-th element of } \mathbf{Y}, \\ 0 & \text { otherwise. }\end{cases}
$$

So $\mathbf{y}_{\mathbf{t}}=\mathbf{S}_{\mathbf{t}} \mathbf{Y}$. By taking the derivatives of Eq. (10) with respect to $t$ and setting it to zero we get

$$
\mathbf{U Y}=\mathbf{V}
$$

where

$$
\mathbf{U}=\sum_{t} \mathbf{S}_{\mathbf{t}}^{T}\left(\mathbf{I}_{\mathbf{t}}-\mathbf{x}_{\mathbf{t}}^{T}\left(\mathbf{x}_{\mathbf{t}} \mathbf{x}_{\mathbf{t}}^{T}+\mathbf{D}_{\mathbf{t}}\right)^{-1} \mathbf{x}_{\mathbf{t}}\right) \mathbf{S}_{\mathbf{t}}
$$

and

$$
\mathbf{V}=\sum_{t} \mathbf{S}_{\mathbf{t}}^{T} \mathbf{x}_{\mathbf{t}}^{T}\left(\mathbf{x}_{\mathbf{t}} \mathbf{x}_{\mathbf{t}}^{T}+\mathbf{D}_{\mathbf{t}}\right)^{-1} \mathbf{D}_{\mathbf{t}} \mathbf{w}_{\mathbf{t}}^{*}
$$

Now we conclude that the optimal compression can be computed by solving a sparse linear Eq. (11). After we get the LDR luminance image $\mathbf{Y}$ in logarithmic domain, we can achieve the LDR luminance image $\mathbf{J}$ in image domain by setting $\mathbf{J}=\exp (\mathbf{Y})$.

As mentioned earlier, our method operates on the input HDR image's luminance channel $\mathbf{I}$. In order to reconstruct the RGB channels, we adopt an approach similar to method of Schlick[21]

$$
\mathbf{J}_{c}=\left(\frac{\mathbf{I}_{c}}{\mathbf{I}}\right)^{s} \mathbf{J} \quad c \in\{r, g, b\},
$$

where $\mathbf{I}_{c}$ and $\mathbf{J}_{c}$ denote one of the RGB color channels before and after tone mapping. The parameter $s$ is the saturation factor. Our results show that $s \in$ $[0.5,0.8]$ works well for most HDR images.

The matrix $\mathbf{U} \in \mathbf{R}^{N \times N}$ in Eq. (12) is symmetric and sparse, and the number of nonzero elements of each row is $(2 \sqrt{K}-1)^{2}$. The computation complexity of constructing matrix $\mathbf{U}$ is about $O\left(N K^{2}\right)$. 


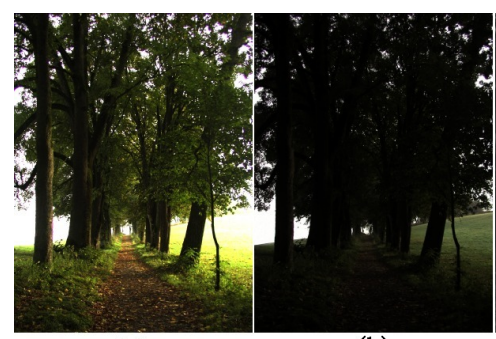

(a)

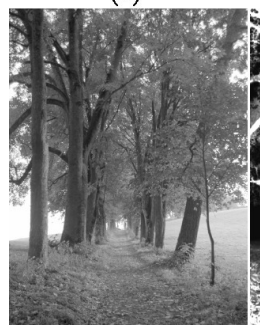

(e) (b)

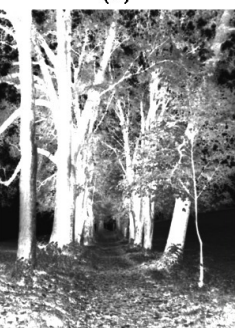

(f)

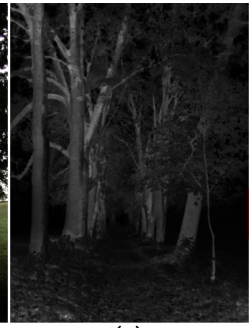

(c)

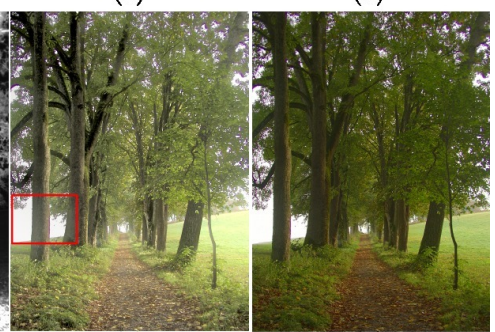

(g)

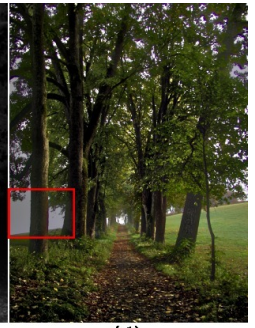

(d)

(h)

Fig. 3. Guided images and its affection. (a) input HDR image. (b) Shan's result without guidance map. (c) Shan's guidance map [8]. (d)Shan's result. (e) our $\alpha$ image. (f) our $\beta$ image. (g) result of local linear model with our two guided images. (h) our result.

\subsection{Guided Image}

In order to guide the modification of local contrast, Shan [8] proposed the concept of guidance map. With the help of guidance map, they can get more satisfactory result, as shown in Figure 3(b), (d). Bright regions in the guidance map indicate that the same areas of the input HDR image should be enhanced, otherwise should be compressed.

However, their approach usually fails when pixel values in a patch is bright enough, as shown in Figure 2(b),(c) and Figure 5(b),(c). Therefore we add a new contraint item $b^{*}$ (namely guided image $\alpha$ ) to constrain the variation of our intercept item $b$. From Figure 3(e), we can see that the guided image $\alpha$ has given a reasonable restriction to our intercept item $b$ which denotes the luminance base. Figure 3(g) is achieved by the locally linear model with our two guided images. Compared with Figure 3(d), Figure 3)(g) is free of the distortions caused by improper intercept item, as shown in sky parts in the rectangle.

A good estimation of guided images becomes very important since they are so critical for a satisfactory result. Fortunately, we find that there are several proper formulations. Following we discuss two essential components in constructing guided images. Since illumination is the main reason of causing the high dynamic range problem, local mean value is needed to estimate the illumination [5]. Local variance is critical to preserve the details because the target of tone mapping is compressing the high dynamic range while preserving the details. If we take these two components into consideration, the explicit formulation of 
guided image is less critical. Finally, we choose the formulation of our two guided images as follows:

$$
\begin{aligned}
w_{t}^{*} & =\frac{1}{u_{t}^{\rho_{1}}+\lambda \sigma_{t}^{\rho_{2}}} . \\
b_{t}^{*} & =u_{t}^{\rho_{3}}+\lambda \sigma_{t}^{\rho_{4}}
\end{aligned}
$$

where $u_{t}$ and $\sigma_{t}$ denotes the mean value and variation of the local patch centering in pixel $t$ respectively. $\lambda=0.1$ balances between the contribution of mean and value and $\rho_{i}(i \in\{1,2,3,4\})$ are parameters which need to be toned.

Compared with the guidance map of Shan [8], our guided image has two advantages. Firstly, our approach is not sensitive to parameters. Default value $\rho_{1}=0.5, \rho_{2}=0.2, \rho_{3}=0.25, \rho_{4}=0.05$ works well for most HDR images. Secondly, our approach can achieve more natural results, as shown in Figure 3)(g). From Figure 3(c),(f), we can find that our guided image is more sensitive to illumination changes, especially at the leaves parts and the path.

\section{Experimental Results}

In our experiments, it takes most of the time to construct the sparse matrix $\mathbf{U}$, similarly with soft matting [20]. Therefore a multigrid method [22] is adopted to accelerate the computation. It takes about 5 seconds to process a $600 \times 800$ pixel image on a PC with a $2.83 \mathrm{GHz}$ Intel Core2 Processor using Matlab. We have tested several window size of $3^{*} 3,5^{*} 5,7^{*} 7,9^{*} 9$ and found that our algorithm was not sensitive to window size. In order to see structures of input HDR images more clearly, most of the input images are enhanced by global linear scaling. The codes of the compared methods are downloaded from their homepage with default parameters recommended by their original authors.

In Figure 4 we compare our approach with three typical global operators and locally linear method [8]. Compared with global linear scaling, global gamma correction can enhance more details. Global gamma correction in logarithmic domain can get a more natural result. However, all these global results are still unpleasing because of losing details or contrast. The locally linear model can get a high contrast result, but it sometimes causes distortions, as shown in red rectangle of Figure 4(e). On the contrary, our method can achieve a natural high contrast result without distortion or halo effects.

Next, we compare our method with locally linear approach in Figure 5. Locally linear method has some distortions in white regions, such as the white area in Figure 2(b), (c) and Figure 5)(c). This kind of distortions, to some extent, can be improved by tuning the parameters. But it is really difficult to find proper parameters which can balance between unveiling the details in dark region and avoiding distortions in bright areas. Locally linear method also fails in patches which contain both dark pixels and bright pixels, for instance the sky-leaves in Figure 3(d). We have further found that local linear model is sensitive to patch size since the luminance value is more likely to vary abruptly in a larger window. Our method does not have these problems. 


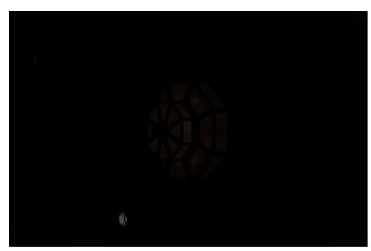

(a)

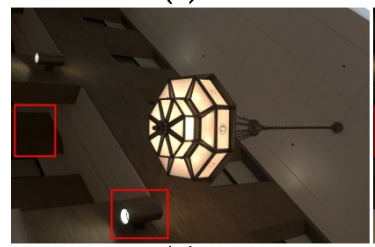

(d)

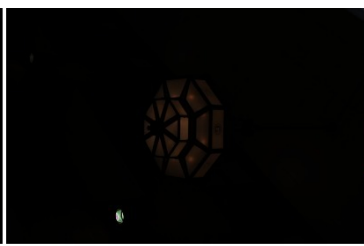

(b)

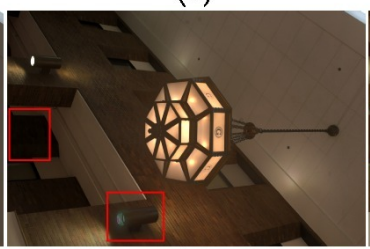

(e)

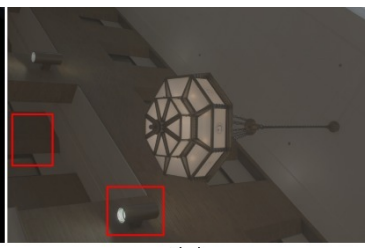

(c)

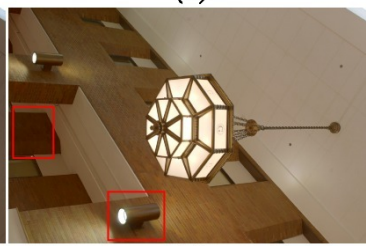

(f)

Fig. 4. Results compared with global operators and locally linear algorithm [8]. (a) input HDR image. (b) result of global linear scaling. (c) result of global gamma correction $(\beta=0.2)$. (d) result of global gamma correction in logarithmic domain $(\beta=0.6)$. (e) result of the locally linear method $\left(\beta_{1}=0.9\right)$ [8] (f) our result. HDR image courtesy of Mark D. Fairchild[23].

In Figure 6] and Figure [7, we compare our method to six state-of-the-art tone mapping operators. Both Durand's Fast Bilateral Filtering method [6] and Farbman's edge-preserving multi-scale decompositions [14] have good performance in terms of preserving the details in bright regions. However, Farbman's method outperforms in details enhancing and details reproduction in dark regions. Compared with these two methods, our operator is better at preserving the details as well as getting a higher contrast, as shown in the statue part in the middle rectangle. Kuang [23] incorporates the spatial processing models in human visual system and propose a new image appearance model which is based on the iCAM framework. Their method does well in some other HDR images, but fails in Figure 6(d). Their result seems a little dim. Locally linear method [8] works quite well in the roof areas but has some distortions in the window parts. Their image has a high contrast but is not good at unveiling the dark regions with the recommended parameters of his paper. Li [2] compresses HDR images with subband architectures and successfully get a quite satisfactory result. Li's subband method can get a high contrast image with precise details in most HDR images. However, in many cases, her method does not enhance details in dark regions very well, as shown in the roof areas in the top rectangle. Figure 7(b) also shows that the red channel of her result is a little abnormal. Paris [3] has proposed an impressive method of tone mapping in terms of details enhancing. Their method also does well in unveiling the dark regions as well as preserving the details in bright areas. Unfortunately, their result does not have a high contrast or a perceptually pleasing appearance. Some distortions can also be found in Figure 7(e). Compared to those state-of-the-art approaches, our approach can effectively compress the dynamic range of the bright areas as well as enhance 


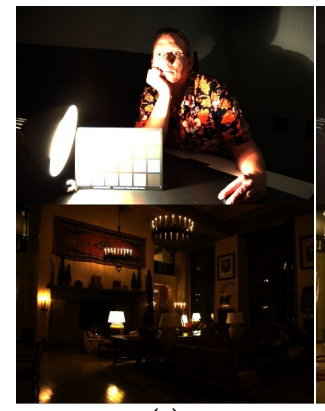

(a)

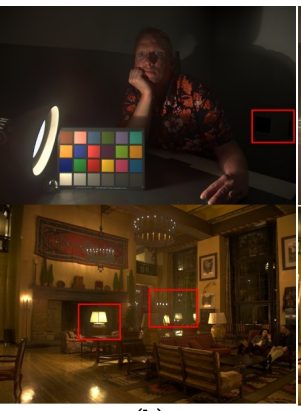

(b)

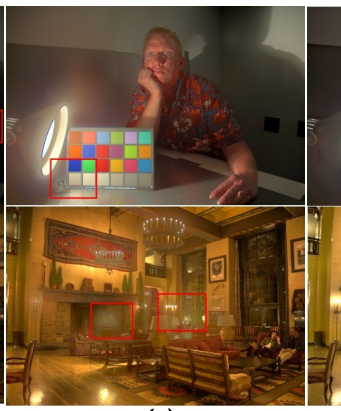

(c)

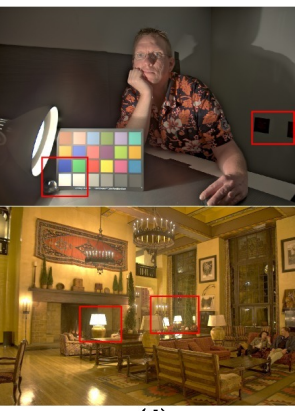

(d)

Fig. 5. Results compared with the locally linear model. (a) input HDR image. (b) the best results of the locally linear model balanced between compression and distortion: $\beta_{1}=0.7$. (c) results of the locally linear model using his recommendatory parameter: $\beta_{1}=0.6, \beta_{2}=0.2, \beta_{3}=0.1$. (d) our result. HDR image courtesy of Mark D. Fairchild [7].

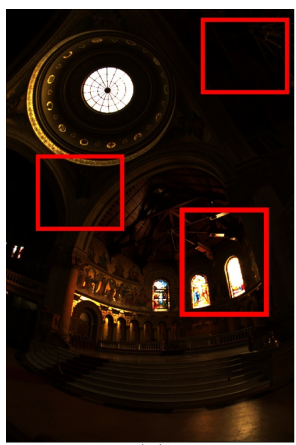

(a)

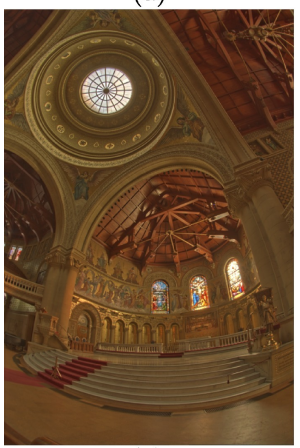

(e)

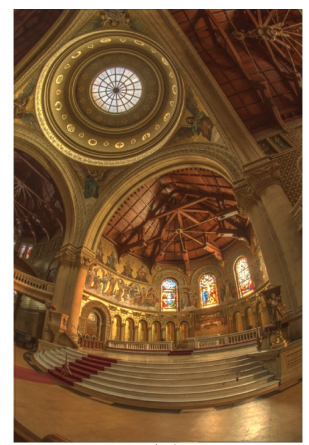

(b)

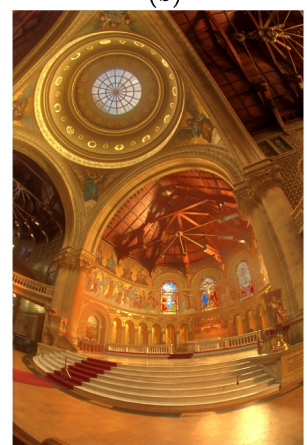

(f)

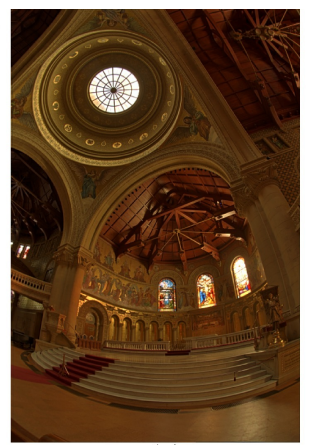

(c)

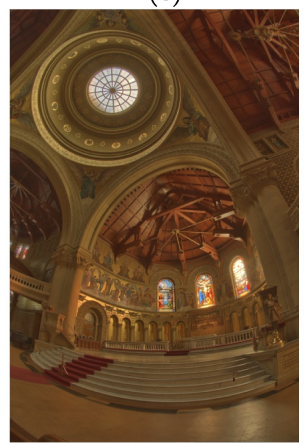

(g)

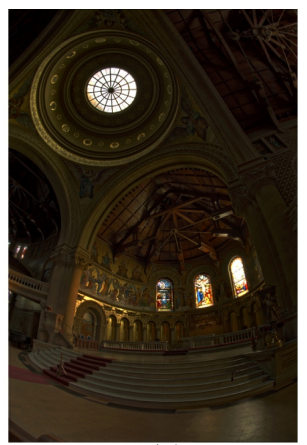

(d)

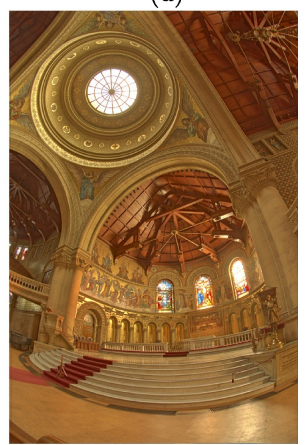

(h)

Fig. 6. Results compared with six state-of-the-art approaches. (a) input HDR image. (b) result of Durand [6]. (c) result of $\mathrm{Li}$ [2]. (d) result of Kuang 23]. (e) result of Farbman [14]. (f) result of $\operatorname{Shan}\left(\beta_{1}=0.6, \beta_{2}=0.2, \beta_{3}=0.1\right)$ [8]. (g) result of Paris [3]. (h) our result. HDR image courtesy of Paul Debevec [4]. 


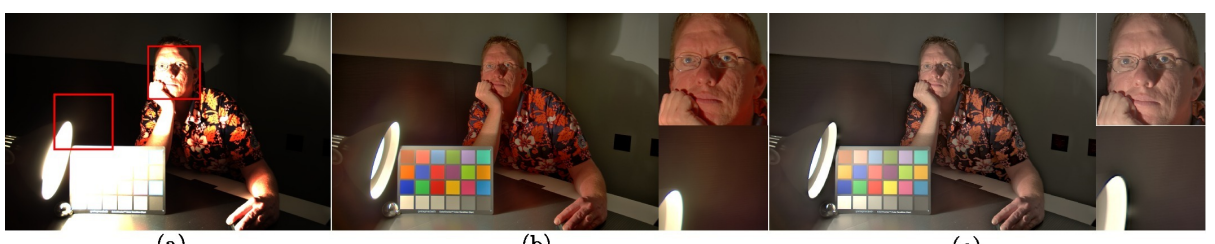

(a)

(b)

(c)

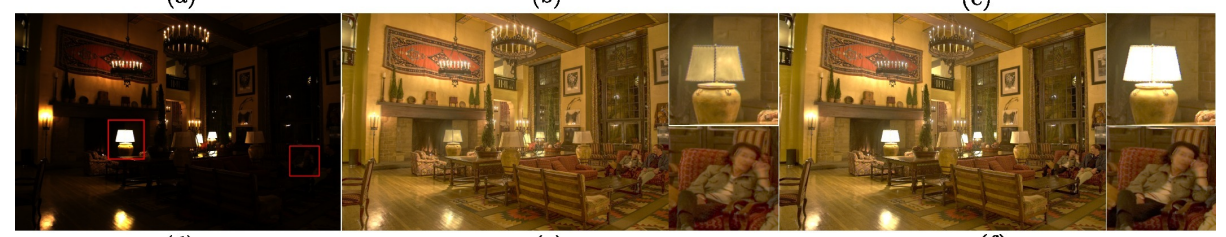

(d)

(e)

(f)

Fig. 7. More comparisons with Li[2] and Paris [3] . (a),(d) input HDR image. (b) result of $\mathrm{Li}$ [2]. (e) result of Paris [3]. (c),(f) our result.

the details in dark regions without distortions or artifacts. From the perceptual perspective, our result has a high contrast and looks natural.

\section{User Study}

Finding whether a tone mapping operator suffers from distortions or halo effects is an easy work. However, it is quite difficult to evaluate whether this tone mapping operator is better than that operator because there is no convincing objective criteria. Fortunately, Yoshida 24] has done a perceptual evaluation of tone mapping operators. Therefore, we designed a similar perceptual evaluation of the above six state-of-the-art tone mapping operators.

The experiment was performed on the Internet with the participation of 23 human observers. The original input HDR image and output LDR images of seven operators were displayed on four web pages. Four perceptually criteria were tested in this experiment, namely naturalness, overall contrast, detail reproduction in dark and bright regions. The observer was asked to vote at most two images to the displayed seven LDR results according to one of the above four criteria. In each web page, eight images were displayed randomly in case of interact. All of the 23 participants were graduate students and researchers of our Lab. None of them were known for the goal of our experiment or tone mapping operators. Table 1 shows the vote results on Figure 6. Due to the limited space, more details about the experiments and more vote results on other tested images will be illustrated in the supplementary.

From Table1, we can find that our method achieved better performances than the state-of-the-art approaches in terms of naturalness and detail reproduction in dark regions. Methods of Shan [8] and Paris [3] did well in detail reproduction in bright regions while Li's approach[2] outperformed in overall contrast. 
Table 1. Perceptual evaluation of the seven tone mapping operators on Figure 6

\begin{tabular}{|c|c|c|c|c|c|c|c|}
\hline Author & Durand & Li & Kuang & Farbman & Shan & Paris & Our \\
\hline Naturalness & 2 & 7 & 1 & 8 & 1 & 4 & $\mathbf{1 5}$ \\
\hline Overall Contrast & 1 & 15 & 0 & 4 & 1 & 4 & $\mathbf{9}$ \\
\hline Details in Dark Regions & 3 & 2 & 2 & 4 & 1 & 8 & $\mathbf{1 2}$ \\
\hline Details in Bright Regions & 6 & 2 & 1 & 3 & 13 & 10 & $\mathbf{1}$ \\
\hline Total Votes & 15 & 26 & 4 & 19 & 16 & 26 & $\mathbf{3 7}$ \\
\hline
\end{tabular}

\section{Conclusions}

In this paper, we have introduced a new local operator for HDR image compression. The main contributions of our work are from two aspects. First, we propose an effective locally nonlinear model-local gamma correction with adaptive parameters. Our model has three properties: reasonable physical explanation, wide applicability and easy implementation. Second, we introduced two constraint items into our energy function and induced a close form solution by solving a sparse linear equation. With two guided images, our algorithm can not only effectively preserve the fine details but also achieve a natural high contrast result without any distortions or halo effects. Comparisons with six state-of-the-art methods have demonstrated that our approach can achieve better performances than the state-of-the-art approaches. Future work will concentrate on expanding our locally nonlinear model and applying it to different possible applications.

Acknowledgments. This work was supported in part by the National Basic Research Program of China under Grant 2012CB316304, and the NSFC under Grant 61175025,61075016.

\section{References}

1. Tumblin, J.E.J.: Three methods of detail-preserving contrast reduction for displayed images. Morgan Kaufmann Publishers Inc., San Francisco (1999)

2. Li, Y., Sharan, L., Adelson, E.H.: Compressing and companding high dynamic range images with subband architectures. ACM Trans. Graph. 24, 836-844 (2005)

3. Paris, S., Hasinoff, S.W., Kautz, J.: Local laplacian filters: edge-aware image processing with a laplacian pyramid. ACM Trans. Graph. (30), 68:1-68:12

4. Debevec, P.E., Malik, J.: Recovering high dynamic range radiance maps from photographs. In: SIGGRAPH 1997, NY, USA, pp. 369-378 (1997)

5. Stockham Jr., T.G.: Image processing in the context of a visual model. Proceedings of the IEEE 60, 828-842 (1972)

6. Durand, F., Dorsey, J.: Fast bilateral filtering for the display of high-dynamic-range images. ACM Trans. Graph. 21, 257-266 (2002)

7. Fairchild, M.D.: The HDR Photographic Survey. MDF Publications, Rochester Institute of Technology, NY, USA (2008) 
8. Shan, Q., Jia, J., Brown, M.S.: Globally optimized linear windowed tone mapping. IEEE Trans. on Visualization and Computer Graphics 16, 663-675 (2010)

9. Drago, F., Myszkowski, K., Annen, T., Chiba, N.: Adaptive logarithmic mapping for displaying high contrast scenes. Computer Graphics Forum 22, 419-426 (2003)

10. Larson, G., Rushmeier, H., Piatko, C.: A visibility matching tone reproduction operator for high dynamic range scenes. IEEE Trans. on Visualization and Computer Graphics 3, 291-306 (1997)

11. Pattanaik, S.N., Tumblin, J., Yee, H., Greenberg, D.P.: Time-dependent visual adaptation for fast realistic image display. In: SIGGRAPH, NY, USA, pp. 47-54 (2000)

12. Qiu, G., Guan, J., Duan, J., Chen, M.: Tone mapping for hdr image using optimization a new closed form solution. In: Proceedings of the 18th International Conference on Pattern Recognition, Washington, DC, USA, pp. 996-999 (2006)

13. Tumblin, J., Rushmeier, H.: Tone reproduction for realistic images. IEEE Comput. Graph. Appl. 13, 42-48 (1993)

14. Farbman, Z., Fattal, R., Lischinski, D., Szeliski, R.: Edge-preserving decompositions for multi-scale tone and detail manipulation. ACM Trans. Graph. 27, 67:167:10 (2008)

15. Fattal, R., Lischinski, D., Werman, M.: Gradient domain high dynamic range compression. ACM Trans. Graph. 21, 249-256 (2002)

16. Lee, J.W., Park, R.H., Chang, S.: Local tone mapping using k-means algorithm and automatic gamma setting. In: IEEE International Conference on Consumer Electronics (ICCE), pp. 807-808 (2011)

17. Wagenaar, W.: Stevens vs fechner: A plea for dismissal of the case. Acta Psychologica 39, 225-235 (1975)

18. Reinhard, E., Ward, G., Pattanaik, S., Debevec, P.: High Dynamic Range Imaging: Acquisition, Display, and Image-Based Lighting. Morgan Kaufmann Publishers Inc., San Francisco (2005)

19. Xiang, S., Nie, F., Pan, C., Zhang, C.: Regression reformulations of lle and ltsa with locally linear transformation. IEEE Transactions on Systems, Man, and Cybernetics, Part B: Cybernetics 41, 1250-1262 (2011)

20. Levin, A., Lischinski, D., Weiss, Y.: A closed-form solution to natural image matting. IEEE Trans. on Pattern Analysis and Machine Intelligence 30, 228-242 (2008)

21. Schlick, C.: A customizable reflectance model for everyday rendering. In: Fourth Eurographics Workshop on Rendering, pp. 73-83 (1993)

22. Hager, W.W., Huang, S.J., Pardalos, P.M., Prokopyev, O.A.: Multiscale Optimization Methods and Applications. Springer (2005)

23. Kuang, J., Johnson, G.M., Fairchild, M.D.: icam06: A refined image appearance model for hdr image rendering. J. Vis. Comun. Image Represent. 18, 406-414 (2007)

24. Yoshida, A., Blanz, V., Myszkowski, K., Peter Seidel, H.: Perceptual evaluation of tone mapping operators with real-world scenes. In: Human Vision and Electronic Imaging X, pp. 192-203. SPIE (2005) 